\title{
Charm mass effects in bulk channel correlations
}

\author{
Y. Burnier ${ }^{a}$ and M. Laine ${ }^{b}$ \\ ${ }^{a}$ Institute of Theoretical Physics, EPFL, \\ CH-1015 Lausanne, Switzerland \\ ${ }^{b}$ Institute for Theoretical Physics, Albert Einstein Center, University of Bern, \\ Sidlerstrasse 5, CH-3012 Bern, Switzerland \\ E-mail: yannis.burnier@epfl.ch, laine@itp.unibe.ch
}

\begin{abstract}
The bulk viscosity of thermalized QCD matter at temperatures above a few hundred $\mathrm{MeV}$ could be significantly influenced by charm quarks because their contribution arises four perturbative orders before purely gluonic effects. In an attempt to clarify the challenges of a lattice study, we determine the relevant imaginary-time correlator (of massive scalar densities) up to NLO in perturbation theory, and compare with existing data. We find discrepancies much larger than in the vector channel; this may hint, apart from the importance of taking a continuum limit, to larger non-perturbative effects in the scalar channel. We also recall how a transport peak related to the scalar density spectral function encodes non-perturbative information concerning the charm quark chemical equilibration rate close to equilibrium.
\end{abstract}

KeYwords: Thermal Field Theory, Quark-Gluon Plasma, Lattice QCD, Heavy Quark Physics

ArXiv ePrint: 1309.1573 


\section{Contents}

1 Introduction 1

2 Physics background $\quad 2$

3 Setup of the computation $\quad 4$

4 Analytic results 5

4.1 Wick contractions 5

$\begin{array}{lll}4.2 & \text { Result after Matsubara sums and angular and partial integrals } & 6\end{array}$

$\begin{array}{lll}4.3 & \text { Infrared and ultraviolet regimes } & 8\end{array}$

5 Numerical evaluations $\quad \mathbf{8}$

5.1 Parameter choices 8

5.2 Normalization to a free correlator $\quad 9$

$\begin{array}{ll}5.3 & \text { Comparison with gluonic effects } \\ & 10\end{array}$

$\begin{array}{ll}5.4 & \text { Normalization to a reconstructed correlator } \\ \end{array}$

6 Conclusions 14

$\begin{array}{ll}\text { A Master sum-integrals } & 15\end{array}$

B Renormalization of the scalar channel correlator $\quad 16$

\section{Introduction}

Viscosities play an important role in the hydrodynamics of finite-size systems, such as those generated in heavy ion collision experiments. In contrast to thermodynamic functions like the pressure or energy density, the dominant contributions to them arise from the slowest (most weakly interacting) processes relevant for equilibrating energy and momentum flows. This may lead to counter-intuitive results; for instance, in cosmology, neutrinos or dark matter particles could play a dominant role for determining viscosities of the cosmic fluid $[1,2]$.

In this paper we are concerned with the bulk viscosity of a QCD plasma similar to that generated in heavy ion collision experiments [3]. Although less prominent than shear viscosity, it also affects the hydrodynamics of the system in an interesting way: indeed the bulk viscosity could grow rapidly as the temperature decreases below the QCD crossover $[4,5]$ and has then been speculated to contribute to "clusterization" [6]-[9] which might be viewed as a thermodynamical precursor to the chemical freezeout process referred to as hadronization. 
The physical processes relevant for the bulk viscosity are those associated with the breaking of scale invariance. We suspect that at temperatures $T \gtrsim 300 \mathrm{MeV}$ a significant contribution may be given by massive quarks, in particular charm quarks. Despite enhancement factors [10] the charm quarks are unlikely to reach chemical equilibrium within the lifetime of heavy ion collisions; however it is expected that they depart from equilibrium on the side of being too many (cf. e.g. ref. [11]). As suggested by experiments $[12,13]$ and theoretical determinations [14]-[18] (similar conclusions have also been reached through phenomenological studies [19]-[23] as well as investigations in other gauge theories [24][26]), they also rapidly equilibrate kinetically. Nevertheless, to the best of our knowledge, their contribution to bulk viscosity has not been studied in detail, ${ }^{1}$ even though it has been stressed that charm quarks may affect a related quantity, the speed of sound, in a substantial way [27].

In this paper, we do not address the bulk viscosity per se; rather, we consider the imaginary-time correlator from which it can be extracted non-perturbatively. Our goal is to compute a mesonic part of this correlator (cf. eq. (3.1)) up to next-to-leading order (NLO) in perturbation theory. Given that thermal perturbation theory in general works better for mesonic correlators than for gluonic ones, and that the existence of a non-zero mass scale brings us further towards the asymptotically free regime, we should expect to find quantitatively accurate results at $T \gtrsim 300 \mathrm{MeV}$, as has previously been demonstrated in the vector channel [28].

The plan of this paper is the following. After elaborating on the general physics of the bulk channel (section 2), the setup of the computation is outlined (section 3) and the main analytic results are presented (section 4). Numerical illustrations and comparisons with non-perturbative data comprise section 5 , whereas section 6 collects together our findings. Two appendices contain various details related to the NLO computation.

\section{Physics background}

Making use of dimensional regularization (the spacetime dimension is denoted by $D=$ $4-2 \epsilon)$ and expressing the QCD action as

$$
S=\int \mathrm{d} t \int \mathrm{d}^{3-2 \epsilon} \mathbf{x}\left\{-\frac{1}{4} F^{a \mu \nu} F_{\mu \nu}^{a}+\bar{\psi}\left(\frac{i}{2} \overleftrightarrow{\not D}-M_{\mathrm{B}}\right) \psi\right\}
$$

the trace of the energy-momentum tensor, $\hat{\theta}^{\mu \nu}$, is $[29,30]$

$$
\hat{\theta}_{\mu}^{\mu}=c_{\theta} \underbrace{g_{\mathrm{B}}^{2} F^{a \mu \nu} F_{\mu \nu}^{a}}_{\equiv \theta}+\bar{\psi} M_{\mathrm{B}} \psi,
$$

where equations of motion were used for the quark fields. Here $c_{\theta}$ is a numerical factor; ${ }^{2}$ $g_{\mathrm{B}}^{2}$ is the bare gauge coupling; and $g^{2}$ is a dimensionless renormalized gauge coupling,

\footnotetext{
${ }^{1}$ In ref. [3] massive quarks were considered but results were only worked out for $M \ll \pi T$.

${ }^{2}$ More precisely, $c_{\theta}=\lim _{\epsilon \rightarrow 0} \frac{D-4}{4 g_{\mathrm{B}}^{2}}=-\frac{b_{0}}{2}-\frac{b_{1} g^{2}}{4}+\ldots, b_{0}=\frac{11 N_{\mathrm{c}}-4 T_{\mathrm{F}}}{3(4 \pi)^{2}}, b_{1}=\frac{34 N_{\mathrm{c}}^{2}-20 N_{\mathrm{c}} T_{\mathrm{F}}-12 C_{\mathrm{F}} T_{\mathrm{F}}}{3(4 \pi)^{4}}$. The usual group theory factors are $N_{\mathrm{c}}=3, C_{\mathrm{F}} \equiv\left(N_{\mathrm{c}}^{2}-1\right) /\left(2 N_{\mathrm{c}}\right)$ and $T_{\mathrm{F}} \equiv N_{\mathrm{f}} / 2$.
} 
evaluated in the $\overline{\mathrm{MS}}$ scheme at the renormalization scale $\bar{\mu}$. The bare mass parameter $M_{\mathrm{B}}$ is assumed to be a diagonal $N_{\mathrm{f}} \times N_{\mathrm{f}}$ matrix.

The trace $\hat{\theta}_{\mu}^{\mu}$ has a non-zero thermal expectation value,

$$
\left\langle\hat{\theta}_{\mu}^{\mu}\right\rangle_{T}=e-3 p=T^{5} \frac{\mathrm{d}}{\mathrm{d} T}\left(\frac{p}{T^{4}}\right),
$$

where $e$ is the energy density, $p$ is the pressure, $T$ is the temperature, and chemical potentials are assumed zero. In a scale-invariant theory, in which $p \propto T^{4}$, this expectation value vanishes. In $\mathrm{QCD}$, in contrast, it is non-zero, both because of dimensional transmutation and because of non-zero mass parameters. The former effect originates from loop corrections, and indeed $e-3 p=\mathcal{O}\left(c_{\theta} g^{4} T^{4}\right)$ in massless QCD at $T \gg 150 \mathrm{MeV}$. In contrast, in the presence of masses, the expectation value is non-zero even in a free theory:

$$
\left\langle\hat{\theta}_{\mu}^{\mu}\right\rangle_{T}=4 N_{\mathrm{c}} \sum_{i=1}^{N_{\mathrm{f}}} M_{i}^{2} \int_{p} \frac{n_{\mathrm{F}}\left(E_{p, i}\right)}{E_{p, i}}+\mathcal{O}\left(g^{2}\right), \quad \int_{p} \equiv \int \frac{\mathrm{d}^{3} \mathbf{p}}{(2 \pi)^{3}}, \quad E_{p, i} \equiv \sqrt{p^{2}+M_{i}^{2}},
$$

where $n_{\mathrm{F}}$ is the Fermi distribution and $M_{i}$ is a renormalized quark mass of flavour $i$. The expectation value in eq. (2.4) vanishes in the chiral limit $M_{i} \ll \pi T$ and, because of Boltzmann suppression, also for large masses, $M_{i} \gg \pi T$. Yet it can give a rather substantial contribution for $M_{i} \sim \pi T$; for temperatures relevant for heavy ion collision experiments, this could be the case with charm quarks [31]-[34], which have a mass $M_{\mathrm{c}}<M_{D^{0}}=1.86 \mathrm{GeV}$.

In the following, we consider 2-point correlation functions of $\hat{\theta}_{\mu}^{\mu}$. Of particular interest is the real-time correlator $(\mathcal{X} \equiv(t, \mathbf{x}))$

$$
\zeta=\frac{1}{9} \lim _{\omega \rightarrow 0^{+}}\left\{\frac{1}{\omega} \int_{\mathcal{X}} e^{i \omega t}\left\langle\frac{1}{2}\left[\hat{\theta}_{\mu}^{\mu}(\mathcal{X}), \hat{\theta}_{\mu}^{\mu}(0)\right]\right\rangle_{T}\right\}
$$

which yields the bulk viscosity. Similarly to the expectation value in eq. (2.3), the bulk viscosity is perturbatively suppressed in massless QCD [3],

$$
\zeta \sim \frac{T^{3}}{g^{4}}\left(\frac{1}{3}-c_{s}^{2}\right)^{2} \sim c_{\theta}^{2} g^{4} T^{3},
$$

where $c_{s}$ is the speed of sound. In a system with vanishing chemical potentials,

$$
\frac{1}{3}-c_{s}^{2}=\frac{T^{3}}{3 p^{\prime \prime}} \frac{\mathrm{d}}{\mathrm{d} T}\left(\frac{p^{\prime}}{T^{3}}\right)
$$

Even though different from $\left\langle\hat{\theta}_{\mu}^{\mu}\right\rangle_{T}$ in eq. (2.3), eq. (2.7) also measures the breaking of scale invariance, and shows a similar parametric behaviour as $\left\langle\hat{\theta}_{\mu}^{\mu}\right\rangle_{T}$. Therefore, we may expect that $\zeta$ is significantly influenced by quark masses.

The argument can be made more precise by relating $\zeta$ to the heavy quark chemical equilibration rate. For $M_{i} \gg \pi T, \hat{\theta}_{\mu}^{\mu}$ of eq. (2.2) is dominated by the same term $\bar{\psi} M_{i} \psi$ that also dominates the heavy quark Hamiltonian. The shape of the corresponding spectral function was discussed in ref. [35]; if we take the limit in eq. (2.5) all the way down to 
frequencies $\omega \lesssim \Gamma_{\text {chem }}$, where $\Gamma_{\text {chem }}$ is the heavy quark chemical equilibration rate, then the heavy quark contribution to the bulk viscosity may be estimated as

$$
\delta \zeta=\frac{1}{18 T} \lim _{\omega \rightarrow 0}\left\{\frac{2 M_{i}^{2} \chi_{f} \Gamma_{\text {chem }}}{\omega^{2}+\Gamma_{\text {chem }}^{2}}\right\}=\frac{M_{i}^{2} \chi_{f}}{9 T \Gamma_{\text {chem }}} .
$$

Here $\chi_{f}$ denotes the heavy flavour susceptibility. Recalling that at weak coupling $[10,28]$

$$
\begin{aligned}
\Gamma_{\text {chem }} & =\frac{g^{4} C_{\mathrm{F}}}{8 \pi M_{i}^{2}}\left(N_{\mathrm{f}}+2 C_{\mathrm{F}}-\frac{N_{\mathrm{c}}}{2}\right)\left(\frac{T M_{i}}{2 \pi}\right)^{\frac{3}{2}} e^{-M_{i} / T}\left(1+\mathcal{O}\left(\frac{T}{M_{i}}, \sqrt{\frac{g^{4} M_{i}}{T}}\right)\right), \\
\chi_{f} & =4 N_{\mathrm{c}}\left(\frac{M_{i} T}{2 \pi}\right)^{\frac{3}{2}} e^{-M_{i} / T}\left(1+\mathcal{O}\left(\frac{T}{M_{i}}, \frac{g^{2} T}{M_{i}}\right)\right),
\end{aligned}
$$

it is seen that $\delta \zeta \sim M_{i}^{4} / g^{4} T$. Therefore $\delta \zeta$ exceeds the gluonic contribution in eq. (2.6) by four perturbative orders, $\mathcal{O}\left(1 / g^{8}\right)$, as is the case also for $0<M_{i} \ll \pi T[3]$.

The purpose of the present study is to consider the imaginary-time correlator corresponding to eq. (2.5):

$$
\left\langle\int_{\mathbf{x}} \hat{\theta}_{\mu}^{\mu}(X) \hat{\theta}_{\mu}^{\mu}(0)\right\rangle_{T}
$$

where $X \equiv(\tau, \mathbf{x}), 0<\tau<1 / T$, and heavy quarks are assumed to be in full equilibrium. ${ }^{3}$ Recalling eq. (2.2), this correlator contains three terms. The 2-point correlator of $c_{\theta} \theta$ has been computed up to NLO in the weak-coupling expansion $[36,37]$ and compared with high-precision lattice simulations [38] (the lattice data contain no continuum extrapolation but are augmented by a tree-level improvement). Here we consider the 2-point correlator of the fermionic part, cf. eq. (3.1), and compare with quenched lattice data from ref. [39] (these data contain neither a continuum extrapolation nor tree-level improvement but have a fairly fine lattice spacing). In addition, independently of numerical data, we estimate within perturbation theory whether the charm contribution could dominate over the purely gluonic one at phenomenologically interesting temperatures.

Returning to bulk viscosity, it should be noted that in a practical heavy ion collision the system has a finite (short) lifetime, which implies that there is a typical frequency $\omega \sim \mathrm{fm} / c$ that can play a role in the hydrodynamical evolution of the system. If the width of the charm quark transport peak is narrower than this $\left(\Gamma_{\text {chem }}<\omega\right)$, then only gluons and light quarks contribute to the bulk viscosity. If $\Gamma_{\text {chem }} \sim \omega$, then heavy quarks should be included but to do this consistently requires going beyond a hydrodynamical description, perhaps by employing kinetic theory. If $\Gamma_{\text {chem }}>\omega$, a hydrodynamical description applies and the heavy quark contribution should be added to the bulk viscosity. In each case the chemical equilibration rate $\Gamma_{\text {chem }}$ is seen to be a fundamental quantity, whose non-perturbative determination as a function of the heavy quark mass would be more than welcome.

\section{Setup of the computation}

For simplicity, we consider a situation in the following in which there is one quenched heavy quark, of bare mass $M_{\mathrm{B}}$ and renormalized mass $M$ (different schemes are specified

\footnotetext{
${ }^{3}$ Given that in real-world heavy ion collisions heavy quarks appear in overabundance, this can in some sense be considered a conservative assumption.
} 
presently), and $N_{\mathrm{f}}$ massless dynamical quarks. (This means that from now on the quarks in section 2 should be thought of as having $N_{\mathrm{f}}+1$ flavours.) The imaginary-time correlator considered is

$$
G_{\mathrm{S}}(\tau) \equiv M_{\mathrm{B}}^{2} \int_{\mathbf{x}}\langle(\bar{\psi} \psi)(\tau, \mathbf{x})(\bar{\psi} \psi)(0, \mathbf{0})\rangle_{T}, \quad 0<\tau<\beta, \quad \beta \equiv \frac{1}{T},
$$

where $\psi$ denotes a single-flavour heavy quark Dirac spinor. Defined this way, the NLO scalar density correlator is finite after mass and gauge coupling renormalization.

We compute the correlator by first determining the corresponding correlator in momentum space, with an external four-momentum

$$
Q \equiv\left(\omega_{n}, \mathbf{0}\right)
$$

where $\omega_{n}$ is a bosonic Matsubara frequency. Denoting that result by $\tilde{G}_{\mathrm{S}}\left(\omega_{n}\right)$, the correlator of eq. (3.1) is obtained from

$$
G_{\mathrm{S}}(\tau)=T \sum_{\omega_{n}} e^{-i \omega_{n} \tau} \tilde{G}_{\mathrm{S}}\left(\omega_{n}\right)
$$

Given that the definition in eq. (3.1) involves a bare parameter, the issue of renormalization and quark mass definitions plays an important role. The bare mass parameter, $M_{\mathrm{B}}^{2}$, can be expressed as $M_{\mathrm{B}}^{2}=M^{2}+\delta M^{2}$, where the choice of $\delta M^{2}$ defines a scheme. We write

$$
\delta M^{2}=-\frac{6 g^{2} C_{\mathrm{F}} M^{2}}{(4 \pi)^{2}}\left(\frac{1}{\epsilon}+\ln \frac{\bar{\mu}^{2}}{M^{2}}+\frac{4}{3}+\delta\right)+\mathcal{O}\left(g^{4}\right),
$$

where $\bar{\mu}$ is the scale parameter of the $\overline{\mathrm{MS}}$ scheme, and terms of $\mathcal{O}(\epsilon)$ were omitted. For $\delta=0, M^{2}$ corresponds to a pole mass, which tends to compactify analytic expressions but is ambiguous on the non-perturbative level and also leads to problems of convergence (see below). If we choose

$$
\delta=-\ln \frac{\bar{\mu}^{2}}{M^{2}}-\frac{4}{3},
$$

then $M^{2}$ stands for the $\overline{\mathrm{MS}}$ mass, which we denote by $m^{2}(\bar{\mu})$; its asymptotic running reads

$$
m(\bar{\mu})=m\left(\bar{\mu}_{\mathrm{ref}}\right)\left[\frac{\ln \left(\bar{\mu}_{\mathrm{ref}} / \Lambda_{\overline{\mathrm{MS}}}\right)}{\ln \left(\bar{\mu} / \Lambda_{\overline{\mathrm{MS}}}\right)}\right]^{\frac{9 C_{\mathrm{F}}}{11 N_{\mathrm{C}}-4 T_{\mathrm{F}}}},
$$

where typically $\bar{\mu}_{\text {ref }} \equiv 2 \mathrm{GeV}$ is chosen [41]. We leave the value of $\delta$ open for the moment.

\section{Analytic results}

\subsection{Wick contractions}

Denoting propagators by

$$
\Delta_{P} \equiv P^{2}+M^{2}
$$

and Matsubara sum-integrals by $\mathfrak{\&}_{\{P\}} \equiv T \sum_{\left\{p_{n}\right\}} \int_{\mathbf{p}}$, where $\{P\}$ stands for fermionic Matsubara momenta, the tree-level correlator reads 
The counterterm contribution is

$$
\chi=-2 N_{\mathrm{c}} M^{2} \oiint_{\{P\}}\left\{-\frac{2}{\Delta_{P}}+\frac{Q^{2}+4 M^{2}}{\Delta_{P} \Delta_{P-Q}}\right\} .
$$

$$
\mathcal{Z}^{*}=-2 N_{\mathrm{c}} \delta M^{2} \sum_{\{P\}}\left\{-\frac{2}{\Delta_{P}}+\frac{2 M^{2}}{\Delta_{P}^{2}}+\frac{Q^{2}+8 M^{2}}{\Delta_{P} \Delta_{P-Q}}-\frac{2 M^{2}\left(Q^{2}+4 M^{2}\right)}{\Delta_{P}^{2} \Delta_{P-Q}}\right\},
$$

whereas the "genuine" 2-loop graphs amount to

$$
\begin{aligned}
& -\frac{D-2}{K^{2} \Delta_{P}^{2}}+\frac{D-2}{\Delta_{P}^{2} \Delta_{P-K}}+\frac{2}{K^{2} \Delta_{P} \Delta_{P-K}}-\frac{4 M^{2}}{K^{2} \Delta_{P}^{2} \Delta_{P-K}} M^{2} \sum_{K\{P\}} \\
& +\frac{(D-2)\left(Q^{2}+4 M^{2}\right)}{K^{2} \Delta_{P}^{2} \Delta_{P-Q}}-\frac{(D-2)\left(Q^{2}+4 M^{2}\right)}{\Delta_{P}^{2} \Delta_{P-K} \Delta_{P-Q}} \\
& -\frac{16 M^{2}-2(D-2) K \cdot Q+4 Q^{2}}{K^{2} \Delta_{P} \Delta_{P-K} \Delta_{P-Q}}+\frac{4 M^{2}\left(Q^{2}+4 M^{2}\right)}{K^{2} \Delta_{P}^{2} \Delta_{P-K} \Delta_{P-Q}} \\
& -\frac{2(D-2) M^{2}+\frac{1}{2}(D-4) Q^{2}}{\Delta_{P} \Delta_{P-K} \Delta_{P-Q} \Delta_{P-K-Q}}+\frac{8 M^{4}+6 M^{2} Q^{2}+Q^{4}}{K^{2} \Delta_{P} \Delta_{P-K} \Delta_{P-Q} \Delta_{P-K-Q}}
\end{aligned}
$$

\subsection{Result after Matsubara sums and angular and partial integrals}

All the Matsubara sums (cf. appendix A) as well as some of the angular integrals appearing in eqs. (4.2)-(4.4) can be carried out analytically; in addition partial integrations permit for simplifications (cf. ref. [40] for the massless case). To display the results, we employ the functions

$$
D_{E_{1} \cdots E_{k}}^{E_{k+1} \cdots E_{n}}(\tau) \equiv \frac{e^{\left(E_{1}+\cdots+E_{k}\right)(\beta-\tau)+\left(E_{k+1}+\cdots+E_{n}\right) \tau}+e^{\left(E_{1}+\cdots+E_{k}\right) \tau+\left(E_{k+1}+\cdots+E_{n}\right)(\beta-\tau)}}{\left[e^{\beta E_{1}} \pm 1\right] \cdots\left[e^{\beta E_{n}} \pm 1\right]},
$$

where the sign in the denominator is chosen according to whether the particle is a boson or a fermion. The energy variables

$$
\epsilon_{k} \equiv|\mathbf{k}|, \quad E_{p} \equiv \sqrt{p^{2}+M^{2}}, \quad E_{p k} \equiv \sqrt{(\mathbf{p}-\mathbf{k})^{2}+M^{2}}, \quad E_{p k}^{ \pm} \equiv \sqrt{(p \pm k)^{2}+M^{2}}
$$

appear frequently, and we denote $D_{2 E_{p}} \equiv D_{E_{p} E_{p}}$. Then the leading-order (LO) result reads

$$
\begin{aligned}
\left.G_{\mathrm{S}}^{\mathrm{LO}}(\tau)\right|_{\tau \text {-dep. }} & =2 N_{\mathrm{c}} M^{2} \int_{p} \frac{p^{2} D_{2 E_{p}}(\tau)}{E_{p}^{2}}, \\
\left.G_{\mathrm{S}}^{\mathrm{LO}}(\tau)\right|_{\text {const. }} & =-4 N_{\mathrm{c}} M^{2} \int_{p} \frac{M^{2} T n_{\mathrm{F}}^{\prime}\left(E_{p}\right)}{E_{p}^{2}},
\end{aligned}
$$

where a $\tau$-independent part stemming from an approximate transport peak in the corresponding spectral function has been separated. Scheme dependence can be expressed as

$$
\left.\frac{\Delta_{\delta} G_{\mathrm{S}}^{\mathrm{NLO}}(\tau)}{4 g^{2} N_{\mathrm{c}} C_{\mathrm{F}} M^{2}}\right|_{\tau \text {-dep. }}=\frac{3 \delta}{32 \pi^{2}} \int_{p} D_{2 E_{p}}(\tau)\left(\frac{5 M^{2}}{E_{p}^{2}}-2\right)
$$




$$
\left.\frac{\Delta_{\delta} G_{\mathrm{S}}^{\mathrm{NLO}}(\tau)}{4 g^{2} N_{\mathrm{c}} C_{\mathrm{F}} M^{2}}\right|_{\text {const. }}=-\frac{3 \delta}{16 \pi^{2}} \int_{p} \frac{M^{2} T n_{\mathrm{F}}^{\prime}\left(E_{p}\right)}{p^{2}}\left(\frac{5 M^{2}}{E_{p}^{2}}-4\right) .
$$

Introducing the shorthand notations

$$
\Delta_{\sigma \tau} \equiv \epsilon_{k}+\sigma E_{p}+\tau E_{p k}, \quad \Delta_{\sigma}=E_{p}+\sigma E_{p k}
$$

the $\tau$-dependent part of the NLO correction reads (after renormalization, cf. appendix B)

$$
\begin{aligned}
& \frac{\left.G_{\mathrm{S}}^{\mathrm{NLO}}\right|_{\tau \text {-dep. }}}{4 g^{2} N_{\mathrm{c}} C_{\mathrm{F}} M^{2}}=\int_{p} \frac{D_{2 E_{p}}(\tau)}{8 \pi^{2}}\left[\frac{M^{2}}{E_{p}^{2}}\left(1+\frac{p}{E_{p}} \ln \frac{E_{p}+p}{E_{p}-p}\right)-1-\left(1-\frac{M^{2}}{E_{p}^{2}}\right) \int_{0}^{\infty} \mathrm{d} k \frac{2 \theta(k)}{k}\right] \\
& +\int_{p, k} \mathbb{P}\left\{\int_{z} \frac{D_{\epsilon_{k} E_{p} E_{p k}}(\tau) M^{2}}{\epsilon_{k} E_{p} E_{p k} \Delta_{+-} \Delta_{-+}}\left[\frac{\epsilon_{k}^{2}+\left(E_{p}+E_{p k}\right)^{2}}{4 M^{2}}+\frac{\Delta_{--}}{\Delta_{++}}-\frac{\epsilon_{k}^{2}}{\Delta_{+-} \Delta_{-+}}+\frac{4 \epsilon_{k}^{2} M^{2}}{\Delta_{++}^{2} \Delta_{+-} \Delta_{-+}}\right]\right. \\
& +\int_{z} \frac{D_{E_{p} E_{p k}}^{\epsilon_{k}}(\tau) M^{2}}{\epsilon_{k} E_{p} E_{p k} \Delta_{+-} \Delta_{-+}}\left[\frac{\epsilon_{k}^{2}+\left(E_{p}+E_{p k}\right)^{2}}{4 M^{2}}+\frac{\Delta_{++}}{\Delta_{--}}-\frac{\epsilon_{k}^{2}}{\Delta_{+-} \Delta_{-+}}+\frac{4 \epsilon_{k}^{2} M^{2}}{\Delta_{--}^{2} \Delta_{+-} \Delta_{-+}}\right] \\
& -\int_{z} \frac{2 D_{\epsilon_{k} E_{p k}}^{E_{p}}(\tau) M^{2}}{\epsilon_{k} E_{p} E_{p k} \Delta_{++} \Delta_{--}}\left[\frac{\epsilon_{k}^{2}+\left(E_{p}-E_{p k}\right)^{2}}{4 M^{2}}+\frac{\Delta_{+-}}{\Delta_{-+}}-\frac{\epsilon_{k}^{2}}{\Delta_{++} \Delta_{--}}+\frac{4 \epsilon_{k}^{2} M^{2}}{\Delta_{-+}^{2} \Delta_{++} \Delta_{--}}\right] \\
& +\frac{D_{2 E_{p}}(\tau)}{2 \epsilon_{k}^{3}}\left(1-\frac{M^{2}}{E_{p}^{2}}\right)\left[1-\frac{E_{p}^{2}\left(E_{p k}^{+}-E_{p k}^{-}\right)-p \epsilon_{k}\left(E_{p k}^{+}+E_{p k}^{-}\right)}{2 p\left(E_{p}^{2}-\epsilon_{k}^{2}\right)}-\frac{\epsilon_{k}^{2} M^{2}\left(E_{p k}^{+}-E_{p k}^{-}\right)}{p\left(E_{p}^{2}-\epsilon_{k}^{2}\right) E_{p k}^{+} E_{p k}^{-}}\right. \\
& \left.-\frac{2 E_{p}^{2}-M^{2}}{2 p E_{p}}\left(\ln \left|\frac{\left(E_{p}+p\right)\left(2 p-\epsilon_{k}\right)}{\left(E_{p}-p\right)\left(2 p+\epsilon_{k}\right)}\right|+\ln \left|\frac{1-\epsilon_{k}^{2} /\left(E_{p}+E_{p k}^{-}\right)^{2}}{1-\epsilon_{k}^{2} /\left(E_{p}+E_{p k}^{+}\right)^{2}}\right|\right)+\theta(k)\right] \\
& +\frac{D_{2 E_{p}}(\tau) n_{\mathrm{B}}\left(\epsilon_{k}\right)}{\epsilon_{k}}\left[-\frac{1}{2 p E_{p}} \ln \frac{E_{p}+p}{E_{p}-p}\right. \\
& \left.+\left(1-\frac{M^{2}}{E_{p}^{2}}\right)\left(\frac{1}{\epsilon_{k}^{2}}-\frac{1}{2 p^{2}}-\frac{2 E_{p}^{2}-M^{2}}{2 p E_{p} \epsilon_{k}^{2}} \ln \frac{E_{p}+p}{E_{p}-p}\right)\right] \\
& +\frac{D_{2 E_{p}}(\tau) n_{\mathrm{F}}\left(E_{k}\right)}{E_{k}}\left[-\frac{1}{2 E_{p}^{2}}-\frac{2 E_{p}^{2}+M^{2}}{2\left(p^{2}-k^{2}\right) E_{p}^{2}}+\frac{M^{2}}{p k E_{p}^{2}} \ln \left|\frac{p+k}{p-k}\right|\right. \\
& \left.\left.+\frac{E_{p}^{2}\left(E_{p}^{2}+E_{k}^{2}-3 M^{2}\right)+M^{4}}{2 p k\left(E_{p}-E_{k}\right) E_{p}^{3}}\left(\ln \left|\frac{p+k}{p-k}\right|+\frac{E_{k}}{E_{p}+E_{k}} \ln \left|\frac{M^{2}+E_{p} E_{k}+p k}{M^{2}+E_{p} E_{k}-p k}\right|\right)\right]\right\},
\end{aligned}
$$

where $\mathbb{P}$ denotes a principal value, $\int_{z}$ an integral over the angles between $\mathbf{p}$ and $\mathbf{k}$ (with $\left.\int_{z} 1=1\right), n_{\mathrm{B}}$ the Bose distribution, and the function $\theta(k)$ is specified in eq. (4.16). The constant contribution reads

$$
\begin{aligned}
& \frac{\left.G_{\mathrm{S}}^{\mathrm{NLO}}\right|_{\text {const. }}}{4 g^{2} N_{\mathrm{c}} C_{\mathrm{F}} M^{2}}=\int_{p} T n_{\mathrm{F}}^{\prime}\left(E_{p}\right) \frac{M^{2}}{E_{p}^{2}}\left\{\frac{3}{4 \pi^{2}}+\int_{k} \frac{n_{\mathrm{B}}\left(\epsilon_{k}\right)}{\epsilon_{k}}\left[\frac{1}{p^{2}}\right]\right. \\
& \left.\quad+\int_{k} \frac{n_{\mathrm{F}}\left(E_{k}\right)}{E_{k}}\left[\frac{1}{p^{2}}\left(1-\frac{M^{2}}{k^{2}}\right)+\frac{1}{k^{2}}+\frac{1}{E_{k}^{2}}+\frac{4 E_{k}^{2}-M^{2}}{2 p k E_{k}^{2}} \ln \left|\frac{p+k}{p-k}\right|\right]\right\} .
\end{aligned}
$$

Note that for $M \gg \pi T$ the leading thermal correction in eq. (4.13), from $\int_{k} n_{\mathrm{B}}\left(\epsilon_{k}\right) / \epsilon_{k}$, agrees with the leading NLO thermal correction to $\chi_{f}[28]$, as is to be expected from integration over the transport peak in eq. (2.8) with the standard relation in eq. (5.16). 


\subsection{Infrared and ultraviolet regimes}

For the numerical evaluation of eq. (4.12) it must be kept in mind that individual parts of the expression contain divergences; only the sum is well-defined. In particular, at small $k$ there is a divergence originating from the terms integrated over $z$ in eq. (4.12) and from terms where the integral had already been carried out. For the latter type the small- $k$ part reads

$$
\frac{D_{2 E_{p}}(\tau)}{\epsilon_{k}^{3}}\left(1-\frac{M^{2}}{E_{p}^{2}}\right)\left(1-\frac{2 E_{p}^{2}-M^{2}}{2 p E_{p}} \ln \frac{E_{p}+p}{E_{p}-p}\right)\left[\frac{1}{2}+n_{\mathrm{B}}\left(\epsilon_{k}\right)\right],
$$

containing both vacuum and Bose-enhanced structures, leading to logarithmic and powerlike divergences. When terms of both origins are summed together, the small- $k$ divergences cancel.

At large $k$ the leading asymptotic behaviour is

$$
\frac{D_{2 E_{p}}(\tau)}{\epsilon_{k}^{3}}\left(1-\frac{M^{2}}{E_{p}^{2}}\right)\left(\frac{3 E_{p}^{2}}{2 \epsilon_{k}^{2}}\right) .
$$

Although integrable this expression is only power-suppressed, and for numerical handling it may be advantageous to accelerate the decrease. The leading tail can be subtracted with the help of the auxiliary function $\theta(k)$ appearing on the 1st and 6th rows of eq. (4.12), for instance

$$
\theta(k) \equiv-\frac{3 E_{p}^{2} \Theta\left(k-k_{\min }\right)}{k^{2}+\lambda^{2}}, \quad \int_{0}^{\infty} \mathrm{d} k \frac{\theta(k)}{k}=-\frac{3 E_{p}^{2}}{2 \lambda^{2}} \ln \left(1+\frac{\lambda^{2}}{k_{\min }^{2}}\right)
$$

The resolution obtained can be increased with a suitable tuning of $\lambda$ and $k_{\min }$; as typical values we have used $\lambda \sim M / 100, k_{\min } \sim 10 T$.

\section{$5 \quad$ Numerical evaluations}

\subsection{Parameter choices}

For a comparison with lattice simulations certain parameter values need to be fixed. Both the gauge coupling and the masses are running parameters; in accordance with ref. [39] we set $\Lambda_{\overline{\mathrm{MS}}} \simeq 216 \mathrm{MeV}$ to fix the gauge coupling (note that the simulations in ref. [39] are for $N_{\mathrm{f}}=0$ ). As a rule the gauge coupling is evolved with 3-loop running (cf. ref. [42] and references therein), with some exceptions as specified below. For the running masses a standard choice according to ref. [41] is to evaluate them at $\bar{\mu}_{\text {ref }}=2 \mathrm{GeV}$, and then the charm mass is $m_{c}\left(\bar{\mu}_{\text {ref }}\right)=1.275(25) \mathrm{GeV}$. However in ref. [39] the simulations correspond to $m_{c}\left(m_{c}\right)=1.094(1) \mathrm{GeV}$, which according to 4-loop evolution [43] as employed in ref. [39] corresponds to $m_{c}\left(\bar{\mu}_{\text {ref }}\right)=0.967(1) \mathrm{GeV}$. (The numbers cited should probably be assigned generous systematic uncertainties.)

As usual, the perturbative predictions display a residual dependence on the $\overline{\mathrm{MS}}$ scale $\bar{\mu}$, which can be used for estimating the uncertainties of a fixed-order computation. We always evaluate the result at some "optimal" scale $\bar{\mu}_{\mathrm{opt}}$ as well as at $0.5 \bar{\mu}_{\mathrm{opt}}$ and $2.0 \bar{\mu}_{\mathrm{opt}}$; the maxima and minima among these three numbers are then used for constructing "error 


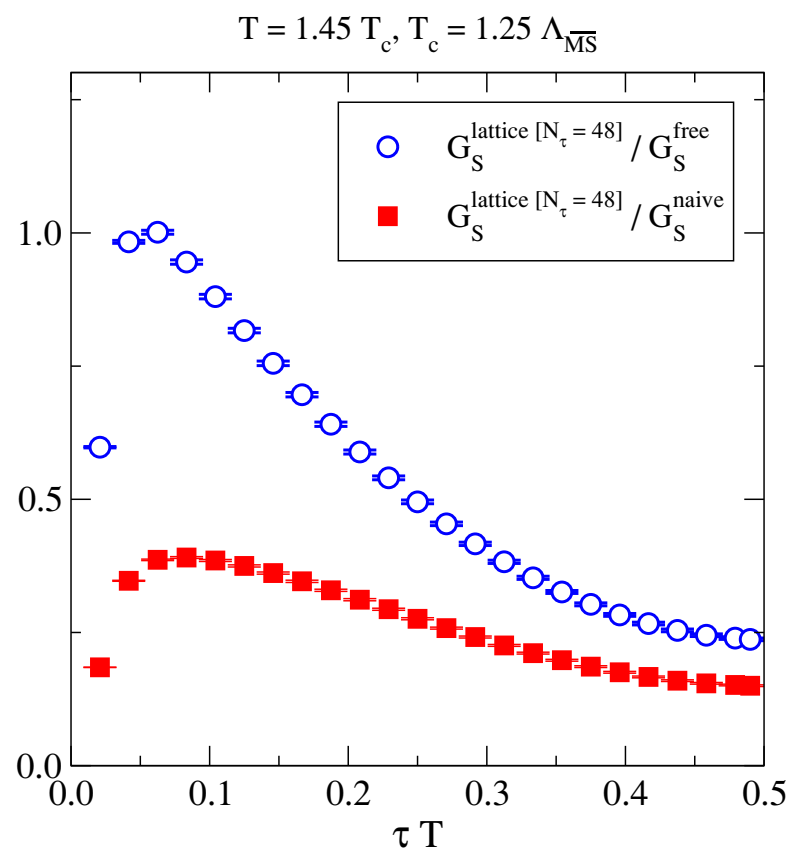

Figure 1. The ratio $G_{\mathrm{S}}^{\text {lattice }} / G_{\mathrm{S}}^{\text {free }}$ (with $m\left(\bar{\mu}_{\text {ref }}\right)=967 \mathrm{MeV}$; open symbols) as well as $G_{\mathrm{S}}^{\text {lattice }} / G_{\mathrm{S}}^{\text {naive }}$ (with $M / T=3.6$ as extracted from a comparison of numerical data and NLO expressions in the vector channel [28]; closed symbols). The lattice data are from ref. [39] and contain no continuum extrapolation. It is seen that normalization to $G_{\mathrm{S}}^{\text {free }}$ (cf. eq. (5.5)) yields results closer to unity at short distances; the very smallest distances are affected by lattice artifacts.

bands" for the perturbative predictions. Obviously such bands only serve as lower bounds for the systematic uncertainties related to the perturbative computation.

In order to display the results in a useful way, they should be normalized to an appropriate expression. We have considered three options for this purpose: a free result with a light quark mass $(M \ll \pi T)$; the scalar correlator in pure Yang-Mills theory; as well as a "reconstructed" correlator following from a zero-temperature spectral function.

\subsection{Normalization to a free correlator}

The LO result for the scalar channel correlator is given in eqs. (4.7), (4.8). It vanishes in the chiral limit, and cannot be evaluated in a closed form for a general mass. Nevertheless, if we assume that $M \ll \pi T$, we can set the mass to zero within the integrand, yielding [44]

$$
G_{\mathrm{S}}^{\text {naive }}(\tau) \equiv N_{\mathrm{c}} T^{3} M^{2}\left[\pi(1-2 \tau T) \frac{1+\cos ^{2}(2 \pi \tau T)}{\sin ^{3}(2 \pi \tau T)}+\frac{2 \cos (2 \pi \tau T)}{\sin ^{2}(2 \pi \tau T)}\right] .
$$

It turns out, however, that this expression does not compare well with lattice data even for $\tau \ll 1 / T$; the situation is illustrated in figure 1 . The reason can be understood as being related to running effects, as we now explain.

Let us start by considering eq. (5.1) at short distances,

$$
G_{\mathrm{S}}^{\text {naive }}(\tau) \stackrel{\tau \ll \frac{1}{T}}{\approx} \frac{N_{\mathrm{c}} M^{2}}{4 \pi^{2} \tau^{3}}
$$


Then compute the NLO correction to this result; making use of the spectral function in eq. (5.13), the $\mathrm{LO}+\mathrm{NLO}$ expression reads

$$
G_{\mathrm{S}}^{\mathrm{LO}+\mathrm{NLO}}(\tau) \stackrel{\tau \ll \frac{1}{M}, \frac{1}{T}}{\approx} \frac{N_{\mathrm{c}} M^{2}}{4 \pi^{2} \tau^{3}}\left[1+\frac{6 g^{2} C_{\mathrm{F}}}{(4 \pi)^{2}}\left(\ln \tau^{2} M^{2}-\delta-\frac{3}{2}+2 \gamma_{\mathrm{E}}\right)\right] .
$$

We note that if $M^{2}$ is kept fixed, $G_{\mathrm{S}}^{\mathrm{LO}+\mathrm{NLO}}$ turns negative at small $\tau$, but that simultaneously the loop expansion breaks down because the NLO correction overtakes the LO term. The problem can be rectified if we use running parameters: going to the $\overline{\mathrm{MS}}$ scheme; choosing $\delta$ according to eq. (3.5) and subsequently replacing $M^{2} \rightarrow m^{2}(\bar{\mu})$ according to eq. (3.6); and taking $\bar{\mu}$ to scale with $\tau$ according to a "fastest apparent convergence" criterion,

$$
\bar{\mu} \rightarrow \frac{\beta e^{\frac{1}{12}-\gamma_{\mathrm{E}}}}{\tau(\beta-\tau)},
$$

such that the NLO correction in eq. (5.3) always remains small, we are led to define

$$
\begin{aligned}
G_{\mathrm{S}}^{\text {free }}(\tau) \equiv & N_{\mathrm{c}} T^{3} m^{2}\left(\bar{\mu}_{\mathrm{ref}}\right)\left\{\frac{\ln \left[\frac{\bar{\mu}_{\mathrm{ref}}}{\Lambda_{\overline{\mathrm{MS}}}}\right]}{\ln \left[\frac{\beta e^{\frac{1}{12}-\gamma_{\mathrm{E}}}}{\tau(\beta-\tau) \Lambda_{\overline{\mathrm{MS}}}}\right]}\right\}^{\frac{18 C_{\mathrm{F}}}{11 N_{\mathrm{c}}-4 T_{\mathrm{F}}}} \\
& \times\left[\pi(1-2 \tau T) \frac{1+\cos ^{2}(2 \pi \tau T)}{\sin ^{3}(2 \pi \tau T)}+\frac{2 \cos (2 \pi \tau T)}{\sin ^{2}(2 \pi \tau T)}\right] .
\end{aligned}
$$

Let us stress that this is a definition rather than an exact result; indeed only the asymptotics at $\tau \ll \beta$ can be fixed unambiguously thanks to asymptotic freedom. Nevertheless, as seen in figure 1, eq. (5.5) agrees much better with lattice data at small distances than eq. (5.1).

The full NLO results normalized to eq. (5.5) are shown in figure 2 as a function of $m\left(\bar{\mu}_{\text {ref }}\right)$. As illustrated with the example of $m\left(\bar{\mu}_{\text {ref }}\right)=0.25 \mathrm{GeV}$, the renormalization scale dependence gets significantly reduced when going from the LO to the NLO level. The NLO results for $m\left(\bar{\mu}_{\text {ref }}\right)=1 \mathrm{GeV}$ agree with the lattice data at small $\tau$, but at large $\tau$ a discrepancy sets in. We return to the discrepancy in connection with figure 3.

\subsection{Comparison with gluonic effects}

The spectral function related to the gluonic part of the trace anomaly, i.e. the operator $c_{\theta} \theta$ defined in eq. (2.2), was computed up to NLO in ref. [37]. At LO it reads (for $\omega \gtrsim \pi T$ )

$$
\rho_{\theta}^{\mathrm{LO}}(\omega)=\frac{N_{\mathrm{c}} C_{\mathrm{F}} c_{\theta}^{2} g^{4} \omega^{4}}{2 \pi}\left[1+2 n_{\mathrm{B}}\left(\frac{\omega}{2}\right)\right]
$$

which yields the correlator

$$
\frac{G_{\theta}^{\text {naive }}(\tau)}{c_{\theta}^{2} g^{4} N_{\mathrm{c}} C_{\mathrm{F}}} \equiv 128 \pi^{2} T^{5}\left[\pi(1-2 \tau T) \frac{2 \cos (2 \pi \tau T)+\cos ^{3}(2 \pi \tau T)}{\sin ^{5}(2 \pi \tau T)}+\frac{1+2 \cos ^{2}(2 \pi \tau T)}{\sin ^{4}(2 \pi \tau T)}\right]
$$

However, there are again loop corrections which imply that the running of the coupling needs to be taken into account; the asymptotics reads [45]

$$
\rho_{\theta}^{\mathrm{vac}}(\omega) \stackrel{\omega \gg \pi T}{\approx} \frac{N_{\mathrm{c}} C_{\mathrm{F}} c_{\theta}^{2} \omega^{4}}{2 \pi}\left\{g^{4}(\bar{\mu})+\frac{g^{6}(\bar{\mu}) N_{\mathrm{c}}}{(4 \pi)^{2}}\left(\frac{22}{3} \ln \frac{\bar{\mu}^{2}}{\omega^{2}}+\frac{73}{3}\right)+\mathcal{O}\left(g^{8}\right)\right\},
$$




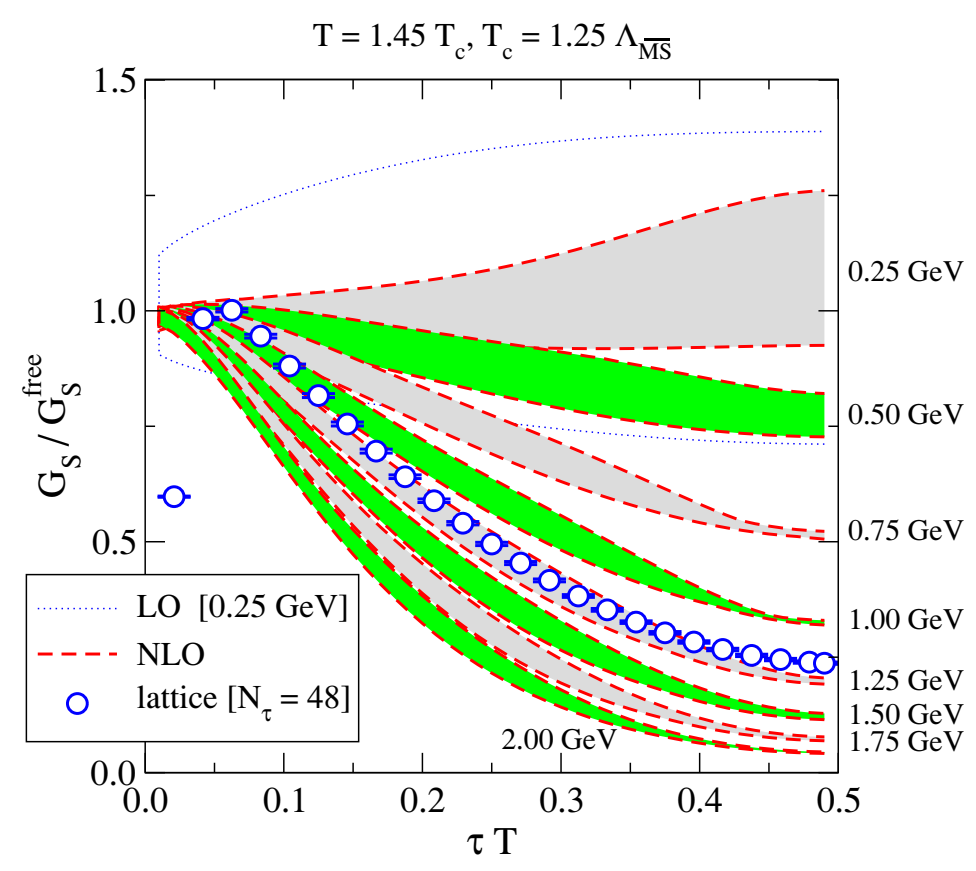

Figure 2. The ratio $G_{\mathrm{S}} / G_{\mathrm{S}}^{\text {free }}$ (cf. eq. (5.5)) as a function of $\tau T$ and the quark mass $m\left(\bar{\mu}_{\text {ref }}\right.$ ), indicated next to the coloured bands. The bands reflect the uncertainty related to the choice of the renormalization scale as specified in the text. The lattice simulations are from ref. [39] and correspond to $m\left(\bar{\mu}_{\text {ref }}\right) \simeq 967 \mathrm{MeV}$. The data at $\tau T>0.15$ are suppressed with respect to the continuum prediction.

with thermal corrections strongly suppressed at $\omega \gg \pi T[36,46]$. Inserting into eq. (5.16) it is seen that at $\tau \ll \beta$ the effects from the running can be captured through a choice analogous to eq. (5.4), viz.

$$
\bar{\mu} \rightarrow \frac{\beta e^{\frac{14}{33}-\gamma_{\mathrm{E}}}}{\tau(\beta-\tau)},
$$

and this leads us to define

$$
\begin{aligned}
G_{\theta}^{\text {free }}(\tau) \equiv & 2 c_{\theta}^{2} N_{\mathrm{c}} C_{\mathrm{F}} T^{5}\left\{\frac{192 \pi^{3}}{\left(11 N_{\mathrm{c}}-4 T_{\mathrm{F}}\right) \ln \left[\frac{\beta e^{\frac{14}{33}-\gamma_{\mathrm{E}}}}{\tau(\beta-\tau) \Lambda_{\overline{\mathrm{MS}}}}\right]}\right\}^{2} \\
& \times\left[\pi(1-2 \tau T) \frac{2 \cos (2 \pi \tau T)+\cos ^{3}(2 \pi \tau T)}{\sin ^{5}(2 \pi \tau T)}+\frac{1+2 \cos ^{2}(2 \pi \tau T)}{\sin ^{4}(2 \pi \tau T)}\right] .
\end{aligned}
$$

Only the asymptotics at $\tau \ll \beta$ is unambiguously fixed, otherwise eq. (5.10) represents a choice. (To be concrete, for this definition we have evaluated the coupling appearing in $c_{\theta}$, cf. footnote 2, at 1-loop level at the scale indicated by eq. (5.9).)

The NLO result for $G_{\mathrm{S}}$, normalized to $G_{\theta}^{\text {free }}$, is shown in figure 3 . With this normalization the agreement at small $\tau$ and the discrepancy at large $\tau$ of the NLO expression and lattice data becomes clearly visible. Independently of lattice data, it is also seen that the relative magnitude of the scalar density correlator in the infrared domain $\tau \sim \beta / 2$ is largest for $m\left(\bar{\mu}_{\text {ref }}\right) \approx 1.0-1.25 \mathrm{GeV}$, quite close to the experimental value 


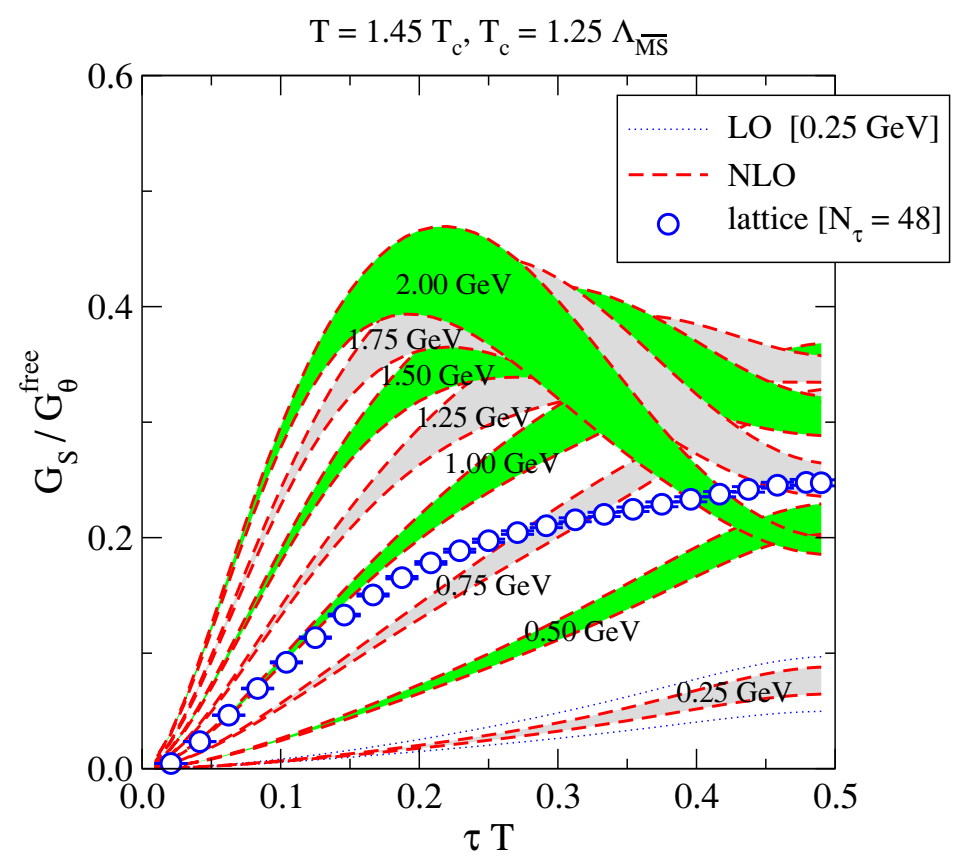

Figure 3. The ratio $G_{\mathrm{S}} / G_{\theta}^{\text {free }}$ (cf. eq. (5.10)) as a function of $\tau T$ and the quark mass $m\left(\bar{\mu}_{\text {ref }}\right.$ ), indicated next to the coloured bands. The bands reflect the uncertainty related to the choice of the renormalization scale. Recalling $m_{c}\left(\bar{\mu}_{\text {ref }}\right)=1.275(25) \mathrm{GeV}[41]$ it is seen that at large distances the charm quark contribution to the bulk channel correlator could be as large as $30-40 \%$; however lattice data [39] at $m\left(\bar{\mu}_{\text {ref }}\right) \simeq 967 \mathrm{MeV}$ are suppressed with respect to the perturbative prediction.

$m_{c}\left(\bar{\mu}_{\text {ref }}\right)=1.275(25) \mathrm{GeV}$ [41]. Whether the relative effect is equally large in $\zeta$ remains to be seen but we nevertheless consider the pattern seen in figure 3 to be intriguing.

\subsection{Normalization to a reconstructed correlator}

On the lattice side it has become fashionable to normalize thermal imaginary-time correlators to a "reconstructed" correlator. Even though we suspect that this increases systematic uncertainties (cf. below), we have worked out this case as well for completeness.

Within perturbation theory, the massive scalar channel spectral function at zero temperature reads (the structure is similar to the vector channel, cf. ref. [47])

$$
\begin{aligned}
& \rho_{\mathrm{S}}^{\mathrm{vac}}(\omega)=\theta(\omega-2 M) \frac{N_{\mathrm{c}} M^{2}\left(\omega^{2}-4 M^{2}\right)^{\frac{3}{2}}}{8 \pi \omega}+\theta(\omega-2 M) \frac{4 g^{2} N_{\mathrm{c}} C_{\mathrm{F}} M^{2}}{(4 \pi)^{3} \omega^{2}}\{ \\
& \left(\omega^{2}-2 M^{2}\right)\left(\omega^{2}-4 M^{2}\right) L_{2}\left(\frac{\omega-\sqrt{\omega^{2}-4 M^{2}}}{\omega+\sqrt{\omega^{2}-4 M^{2}}}\right)+\left(\frac{3}{2} \omega^{4}-2 \omega^{2} M^{2}-13 M^{4}\right) \operatorname{acosh}\left(\frac{\omega}{2 M}\right) \\
& \left.-\omega\left(\omega^{2}-4 M^{2}\right)^{\frac{1}{2}}\left[\left(\omega^{2}-4 M^{2}\right)\left(\ln \frac{\omega\left(\omega^{2}-4 M^{2}\right)}{M^{3}}+\frac{3}{4} \delta\right)-\frac{3}{8}\left(3 \omega^{2}-14 M^{2}\right)\right]\right\}+\mathcal{O}\left(g^{4}\right),
\end{aligned}
$$

where the function $L_{2}$ is defined as

$$
L_{2}(x) \equiv 4 \operatorname{Li}_{2}(x)+2 \operatorname{Li}_{2}(-x)+[2 \ln (1-x)+\ln (1+x)] \ln x .
$$


The ultraviolet asymptotics from here is

$$
\rho_{\mathrm{S}}^{\mathrm{vac}}(\omega) \stackrel{\omega \gg M}{\approx} \frac{N_{\mathrm{c}} \omega^{2} M^{2}}{8 \pi}\left\{1+\frac{6 g^{2} C_{\mathrm{F}}}{(4 \pi)^{2}}\left(\ln \frac{M^{2}}{\omega^{2}}-\delta+\frac{3}{2}\right)+\mathcal{O}\left(g^{4}\right)\right\} .
$$

We note again that in the pole mass scheme, where $M$ is constant and $\delta=0$, eq. (5.13) turns negative at very large $\omega$ and the NLO correction overtakes the LO term, implying a breakdown of the perturbative series. If, however, we go to the $\overline{\mathrm{MS}}$ scheme, choosing $\delta$ according to eq. (3.5) and setting $M^{2} \rightarrow m^{2}(\bar{\mu})$ according to eq. (3.6), we obtain another representation:

$$
\begin{aligned}
\rho_{\mathrm{S}}^{\mathrm{vac}}(\omega) \stackrel{\omega \gg M}{\approx} & \frac{N_{\mathrm{c}} \omega^{2} m^{2}\left(\bar{\mu}_{\mathrm{ref}}\right)}{8 \pi}\left[\frac{\ln \left(\bar{\mu}_{\mathrm{ref}} / \Lambda_{\overline{\mathrm{MS}}}\right)}{\ln \left(\bar{\mu} / \Lambda_{\overline{\mathrm{MS}}}\right)}\right]^{\frac{18 C_{\mathrm{F}}}{11 N_{\mathrm{c}}-4 T_{\mathrm{F}}}} \\
& \times\left\{1+\frac{6 g^{2}(\bar{\mu}) C_{\mathrm{F}}}{(4 \pi)^{2}}\left(\ln \frac{\bar{\mu}^{2}}{\omega^{2}}+\frac{17}{6}\right)+\mathcal{O}\left(g^{4}\right)\right\} .
\end{aligned}
$$

Taking $\bar{\mu}$ to scale with $\omega$, a series is obtained which becomes increasingly convergent as $\omega$ grows. The result is independent of the precise choice of $\bar{\mu}$ up to higher-order corrections; in practice we set $^{4}$

$$
\bar{\mu} \rightarrow \max \left(\pi T, \omega e^{-17 / 12}\right) .
$$

Subsequently the "reconstructed" correlator is defined by taking the vacuum spectral function and using it to compute a thermal Euclidean correlator from

$$
G_{\mathrm{S}}^{\mathrm{rec}}(\tau) \equiv \int_{0}^{\infty} \frac{\mathrm{d} \omega}{\pi} \rho_{\mathrm{S}}^{\mathrm{vac}}(\omega) \frac{\cosh \left(\frac{\beta}{2}-\tau\right) \omega}{\sinh \frac{\beta \omega}{2}} .
$$

In figure 4 results normalized to the reconstructed correlator are shown. As expected, the results approach unity at short distances for all masses (the small- $\tau$ result is determined by large $\omega$ and then thermal corrections are strongly suppressed with respect to the vacuum term $[46,48])$. However the match to lattice data is not as good as in figures 2, 3; among possible explanations we can envisage the following:

- On the continuum side it is questionable whether perturbation theory, which misses all resonance contributions, can reflect the vacuum scalar spectral function even qualitatively in the $\omega$-range dominating eq. (5.16) at moderate $\tau, \omega \gtrsim \pi T \sim 1 \mathrm{GeV}$.

- On the lattice side the theoretical formulae (e.g. eq. (5.16)) used for relating measured data to the reconstructed correlator assume a continuous $\tau$-variable; this necessitates taking a continuum limit which has not been reached.

- The "reconstructed" correlator of ref. [39] was not measured at $T=0$ but at $T=0.73 T_{\mathrm{c}}$.

Because of these issues we refrain from speculating on the origins of the discrepancy in figure 4.

\footnotetext{
${ }^{4}$ The infrared cutoff $\pi T$ only affects the smallest masses, $m\left(\bar{\mu}_{\text {ref }}\right) \lesssim 2 \pi T$.
} 


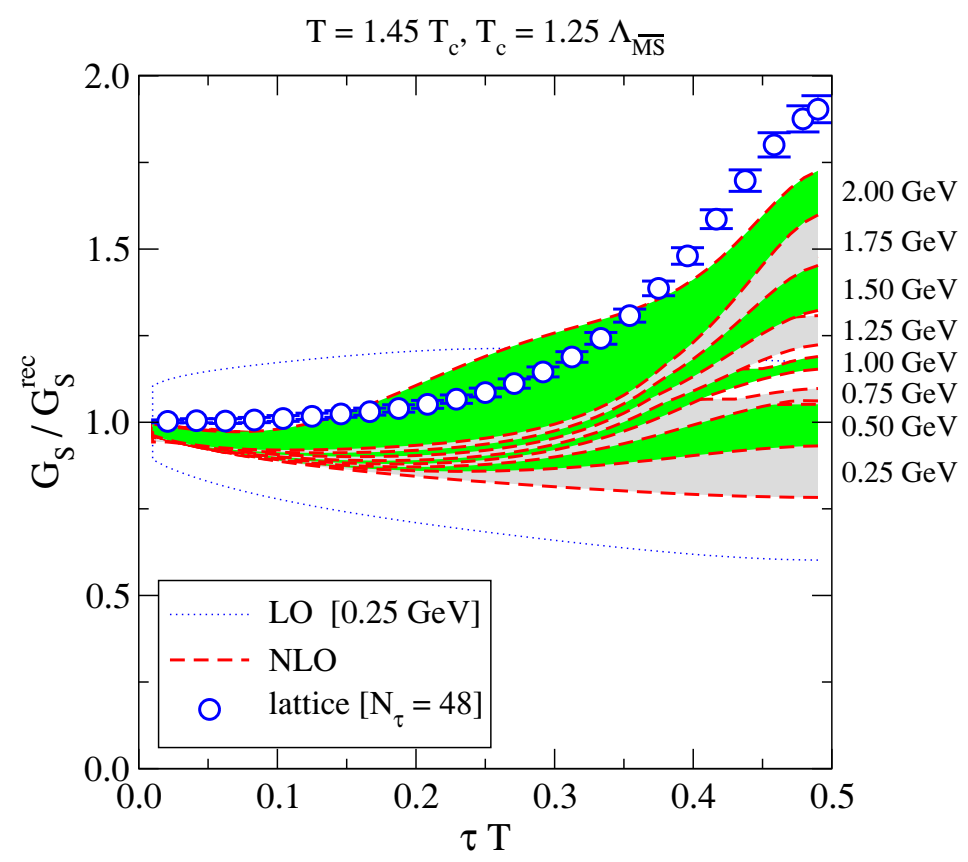

Figure 4. The ratio $G_{\mathrm{S}} / G_{\mathrm{S}}^{\text {rec }}$ (cf. eq. (5.16)) as a function of $\tau T$ and the quark mass $m\left(\bar{\mu}_{\text {ref }}\right)$, indicated next to the coloured bands. The bands reflect the uncertainty related to the choice of the renormalization scale. The lattice simulations are from ref. [39]. The comparison contains uncontrolled uncertainties from both sides as discussed in the text.

\section{Conclusions}

The purpose of this paper has been to investigate the influence of a finite charm quark mass on a 2-point imaginary-time correlator in the so-called bulk (or scalar) channel, corresponding to the trace of the energy-momentum tensor in continuum QCD. We could have anticipated a rather substantial influence, given that in massless QCD the corresponding correlator is non-zero only because of an "anomalous" breaking of scale invariance, and is therefore proportional to a high power of running coupling constants (cf. eq. (5.6)).

Remarkably, the relative influence of a massive quark on the scalar channel correlator at large imaginary-time separations appears to peak for $m\left(\bar{\mu}_{\text {ref }}\right)=1.0-1.25 \mathrm{GeV}$ (cf. figure 3), not far from the physical charm mass $m_{c}\left(\bar{\mu}_{\text {ref }}\right)=1.275(25) \mathrm{GeV}$ [41]. Hopefully this observation motivates a perturbative determination of the bulk viscosity $\zeta$ for all quark masses as well as refined lattice investigations of scalar correlators in the future, including a systematic approach to the continuum limit.

An interesting principal application of the scalar correlator is that it can be used for determining the charm quark chemical equilibration rate close to equilibrium [35], a quantity that experiences a non-trivial (Sommerfeld) enhancement even deep in the non-relativistic regime [10]. The determination poses however a numerical challenge because physics resides in a very narrow transport peak which is difficult to resolve from Euclidean data. (For $M \gg \pi T$ the peak is exponentially narrow, cf. eqs. (2.8), (2.9), rather than only $1 / M$ suppressed like in the vector channel [49]; however for $m\left(\bar{\mu}_{\text {ref }}\right) \sim 1 \mathrm{GeV}$ and a temperature 
$T \gtrsim 400 \mathrm{MeV}$ the difference might not be dramatic. Note also that there are significant challenges in resolving the transport peak even in pure Yang-Mills theory [4], however with connected mesonic correlators a higher numerical precision can typically be reached.)

Taking the current lattice data at face value, we find discrepancies much larger than in the vector channel where an identical data set was used (cf. figures 2, 3 vs. ref. [28]). Part of the reason is surely that the quark mass, with associated uncertainties in its determination, now plays a more prominent role. In order to get the uncertainties under control a continuum limit needs to be taken. Nevertheless it is also tempting to speculate that the scalar channel might experience larger non-perturbative effects than the vector one. Of course, these may partly originate from quarkonium physics rather than the transport regime, cf. e.g. ref. [39] and references therein.

For the full trace of eq. (2.2), an open challenge both on the lattice and in continuum is the study of the "mixed" channel, with correlators involving crossterms between mesonic and purely gluonic operators. On the lattice this correlator might be rather noisy numerically, but hopefully suitable methods will eventually be developed. A similar problem concerns the disconnected contraction of mesonic scalar densities.

It may finally be wondered why we have concentrated on the bulk rather than the more prominent shear channel. The reason is that for bulk viscosity the effects from massless QCD are "anomalously" suppressed, so that the contribution from the explicit breaking of scale invariance through quark masses is relatively speaking more important. In the shear channel the charm mass also has an influence, however we do not expect it to be larger than $\sim 10 \%$ as has been observed in basic thermodynamic quantities at $T \gtrsim 400 \mathrm{MeV}$ before [31]-[34].

Note added. We have recently explored possible reasons for the discrepancy between NLO expressions and lattice data in the scalar channel, by varying the corresponding spectral function, and found that the discrepancy is likely to originate from quarkonium-related physics, more precisely from the proper location of the quark-antiquark threshold [50].

\section{Acknowledgments}

We thank H.-T. Ding for helpful discussions and providing us with lattice data from ref. [39], and G.D. Moore for helpful discussions. This work was partly supported by the Swiss National Science Foundation (SNF) under the grant 200021-140234 as well as under the Ambizione grant PZ00P2-142524.

\section{A Master sum-integrals}

The way that the computation is organized is by first using substitutions of sum-integration variables in order to express the result in terms of a small number of "master" sum-integrals, or basis functions, cf. eq. (4.4). After transformation to coordinate space the basis functions 
can be defined as

$$
\left.\mathcal{I}_{n_{1} n_{2} n_{3} n_{4} n_{5}}^{m_{1} m_{2} m_{3}}(\tau) \equiv T \sum_{\omega_{n}} e^{-i \omega_{n} \tau} \sum_{K\{P\}} \frac{\left(M^{2}\right)^{m_{1}}\left(Q^{2}\right)^{m_{2}}(2 K \cdot Q)^{m_{3}}}{\left(K^{2}\right)^{n_{1}} \Delta_{P}^{n_{2}} \Delta_{P-K}^{n_{3}} \Delta_{P-Q}^{n_{4}} \Delta_{P-K-Q}^{n_{5}}}\right|_{Q=\left(\omega_{n}, \mathbf{0}\right)} .
$$

In ref. [28] expressions obtained after carrying out the Matsubara sums were given for all the basis functions appearing in the present computation, and these have been employed in order to arrive at eqs. (B.1), (B.2) below. Here we just remark, for completeness, that one of the basis functions discussed in ref. [28] is actually not independent of the others:

$$
\mathcal{I}_{-11111}^{000}(\tau)=2 \mathcal{I}_{01110}^{000}(\tau)-2 \mathcal{I}_{01111}^{100}(\tau)-\frac{1}{2} \mathcal{I}_{01111}^{010}(\tau)
$$

\section{B Renormalization of the scalar channel correlator}

Expressing eq. (4.4) in terms of the basis functions listed in appendix A of ref. [28], we obtain results for the scalar correlator in which the Matsubara sums have been carried out:

$$
\begin{aligned}
& \frac{\left.G_{\mathrm{S}}^{\mathrm{NLO}}\right|_{\tau-\text { dep. }}}{4 g^{2} N_{\mathrm{c}} C_{\mathrm{F}} M^{2}} \\
& =\int_{\mathbf{p}, \mathbf{k}} \frac{D_{\epsilon_{k} E_{p} E_{p k}}(\tau) M^{2}}{\epsilon_{k} E_{p} E_{p k} \Delta_{+-} \Delta_{-+}}\left[\frac{\epsilon_{k}^{2}+\left(E_{p}+E_{p k}\right)^{2}}{4 M^{2}}+\frac{\Delta_{--}}{\Delta_{++}}-\frac{\epsilon_{k}^{2}}{\Delta_{+-} \Delta_{-+}}+\frac{4 \epsilon_{k}^{2} M^{2}}{\Delta_{++}^{2} \Delta_{+-} \Delta_{-+}}\right] \\
& +\int_{\mathbf{p}, \mathbf{k}} \frac{D_{E_{p} E_{p k}}^{\epsilon_{k}}(\tau) M^{2}}{\epsilon_{k} E_{p} E_{p k} \Delta_{+-} \Delta_{-+}}\left[\frac{\epsilon_{k}^{2}+\left(E_{p}+E_{p k}\right)^{2}}{4 M^{2}}+\frac{\Delta_{++}}{\Delta_{--}}-\frac{\epsilon_{k}^{2}}{\Delta_{+-} \Delta_{-+}}+\frac{4 \epsilon_{k}^{2} M^{2}}{\Delta_{--}^{2} \Delta_{+-} \Delta_{-+}}\right] \\
& -\int_{\mathbf{p}, \mathbf{k}} \frac{2 D_{\epsilon_{k} E_{p k}}^{E_{p}}(\tau) M^{2}}{\epsilon_{k} E_{p} E_{p k} \Delta_{++} \Delta_{--}}\left[\frac{\epsilon_{k}^{2}+\left(E_{p}-E_{p k}\right)^{2}}{4 M^{2}}+\frac{\Delta_{+-}}{\Delta_{-+}}-\frac{\epsilon_{k}^{2}}{\Delta_{++} \Delta_{--}}+\frac{4 \epsilon_{k}^{2} M^{2}}{\Delta_{-+}^{2} \Delta_{++} \Delta_{--}}\right] \\
& +\int_{\mathbf{p}} D_{2 E_{p}}(\tau)\{\text { “eq. (B.3)" } \\
& +\int_{\mathbf{k}} \frac{n_{\mathrm{B}}\left(\epsilon_{k}\right)}{\epsilon_{k}}\left[\frac{M^{2}}{E_{p}^{4}}-\frac{M^{2}\left(E_{p}^{2}-M^{2}\right)}{2 E_{p}^{3} E_{p k}}\left(\frac{1}{\Delta_{++}^{2}}+\frac{1}{\Delta_{--}^{2}}-\frac{1}{\Delta_{+-}^{2}}-\frac{1}{\Delta_{-+}^{2}}\right)\right. \\
& +\frac{E_{p}^{2}+E_{p k}^{2}-M^{2}}{E_{p} E_{p k}}\left(\frac{1}{\Delta_{++} \Delta_{--}}-\frac{1}{\Delta_{+-} \Delta_{-+}}\right) \\
& \left.+\frac{M^{2}\left(2 E_{p}^{2}-M^{2}\right)}{E_{p}^{4}}\left(\frac{1}{\Delta_{++} \Delta_{--}}+\frac{1}{\Delta_{+-} \Delta_{-+}}\right)\right] \\
& +\int_{\mathbf{k}} \frac{n_{\mathrm{F}}\left(E_{p k}\right)}{E_{p k}} \mathbb{P}\left[\frac{M^{2}}{E_{p}^{4}}-\frac{M^{2}\left(E_{p}^{2}-M^{2}\right)}{2 \epsilon_{k} E_{p}^{3}}\left(\frac{1}{\Delta_{-+}^{2}}+\frac{1}{\Delta_{--}^{2}}-\frac{1}{\Delta_{+-}^{2}}-\frac{1}{\Delta_{++}^{2}}\right)\right. \\
& +\frac{E_{p}^{2}+E_{p k}^{2}-M^{2}}{E_{p}}\left(\frac{1}{\Delta_{+} \Delta_{++} \Delta_{--}}+\frac{1}{\Delta_{-} \Delta_{+-} \Delta_{-+}}\right)-\frac{M^{2}}{E_{p}^{2} \Delta_{+} \Delta_{-}} \\
& \left.\left.+\frac{E_{p k} M^{2}\left(2 E_{p}^{2}-M^{2}\right)}{E_{p}^{4}}\left(\frac{1}{\Delta_{+} \Delta_{++} \Delta_{--}}-\frac{1}{\Delta_{-} \Delta_{+-} \Delta_{-+}}\right)\right]\right\} \\
& +\int_{\mathbf{p}} E_{p} \partial_{E_{p}} D_{2 E_{p}}(\tau)\{0
\end{aligned}
$$




$$
\begin{aligned}
& +\int_{\mathbf{k}} \frac{n_{\mathrm{B}}\left(\epsilon_{k}\right)}{\epsilon_{k}}\left[\frac{1}{2 E_{p}^{2}}-\frac{M^{2}}{2 E_{p}^{4}}-\frac{M^{2}\left(E_{p}^{2}-M^{2}\right)}{2 E_{p}^{4}}\left(\frac{1}{\Delta_{++} \Delta_{+-}}+\frac{1}{\Delta_{--} \Delta_{-+}}\right)\right] \\
& \left.+\int_{\mathbf{k}} \frac{n_{\mathrm{F}}\left(E_{p k}\right)}{E_{p k}}\left[\frac{1}{2 E_{p}^{2}}-\frac{M^{2}}{2 E_{p}^{4}}-\frac{M^{2}\left(E_{p}^{2}-M^{2}\right)}{2 E_{p}^{4}}\left(\frac{1}{\Delta_{++} \Delta_{--}}+\frac{1}{\Delta_{+-} \Delta_{-+}}\right)\right]\right\} .
\end{aligned}
$$

The constant contribution, in turn, can be expressed as

$$
\begin{aligned}
& \frac{\left.G_{\mathrm{S}}^{\mathrm{NLO}}\right|_{\text {const. }}}{4 g^{2} N_{\mathrm{c}} C_{\mathrm{F}} M^{2}} \\
& =\int_{\mathbf{p}, \mathbf{k}} \frac{T n_{\mathrm{F}}^{\prime}\left(E_{p}\right) n_{\mathrm{F}}^{\prime}\left(E_{p k}\right)}{2 E_{p}^{2} E_{p k}^{2}}\left\{-2 M^{2}+2 M^{4}\left(\frac{1}{\Delta_{++} \Delta_{--}}+\frac{1}{\Delta_{+-} \Delta_{-+}}\right)\right\} \\
& +\int_{\mathbf{p}} 2 T n_{\mathrm{F}}^{\prime}\left(E_{p}\right)\{\text { "eq. (B.4)" } \\
& \quad+\int_{\mathbf{k}} \frac{n_{\mathrm{B}}\left(\epsilon_{k}\right)}{\epsilon_{k}}\left[\frac{M^{2}}{E_{p}^{4}}-\frac{2 \epsilon_{k} M^{4}}{E_{p}^{3}}\left(\frac{1}{\Delta_{++}^{2} \Delta_{+-}^{2}}-\frac{1}{\Delta_{--}^{2} \Delta_{-+}^{2}}\right)\right. \\
& \left.\quad+\frac{M^{2}\left(2 E_{p}^{2}-M^{2}\right)}{E_{p}^{4}}\left(\frac{1}{\Delta_{++} \Delta_{+-}}+\frac{1}{\Delta_{--} \Delta_{-+}}\right)\right] \\
& \quad+\int_{\mathbf{k}} \frac{n_{\mathrm{F}}\left(E_{p k}\right)}{E_{p k}}\left[\frac{M^{2}}{E_{p}^{4}}+\frac{M^{2}}{E_{p}^{2} E_{p k}^{2}}+\frac{2 M^{4}}{E_{p}^{3} E_{p k}}\left(\frac{\Delta_{+}^{2}}{\Delta_{++}^{2} \Delta_{--}^{2}}-\frac{\Delta_{-}^{2}}{\Delta_{+-}^{2} \Delta_{-+}^{2}}\right)\right. \\
& +\int_{\mathbf{p}} 2 T E_{p} n_{\mathrm{F}}^{\prime \prime}\left(E_{p}\right)\{0 \\
& \left.\quad+\int_{\mathbf{k}} \frac{n_{\mathrm{B}}\left(\epsilon_{k}\right)}{\epsilon_{k}}\left[-\frac{M^{2}}{2 E_{p}^{4}}+\frac{M^{4}}{2 E_{p}^{4}}\left(\frac{1}{E_{p}^{2} E_{p k}^{2}}\right)\left(\frac{1}{\Delta_{++} \Delta_{--}}+\frac{1}{\Delta_{+-} \Delta_{-+}}\right)\right]\right\} \\
& \left.\quad+\int_{\mathbf{k}} \frac{n_{\mathrm{F}}\left(E_{p k}\right)}{E_{p k}}\left[-\frac{M^{2}}{2 E_{p}^{4}}+\frac{M^{4}}{2 E_{p}^{4}}\left(\frac{1}{\Delta_{++} \Delta_{--}}+\frac{1}{\Delta_{+--} \Delta_{-+}}\right)\right]\right\} .
\end{aligned}
$$

The "0"s in eqs. (B.1), (B.2) represent vacuum contributions that vanish after renormalization. The coefficients of $D_{2 E_{p}}(\tau)$ and $T n_{\mathrm{F}}^{\prime}\left(E_{p}\right)$ are also related to renormalization, but do not vanish:

$$
\begin{aligned}
\text { "eq. (B.3)" }= & \int_{\mathbf{k}} \mathbb{P}\left\{\frac{(1-\epsilon)\left(2 M^{2}-E_{p}^{2}\right)}{2 E_{p}^{2} M^{2}}\left(\frac{1}{\epsilon_{k}}-\frac{1}{E_{p k}}\right)-\frac{\epsilon E_{p}^{2}+(1-\epsilon) M^{2}}{2 E_{p}^{2} E_{p k}\left(E_{p k}^{2}-E_{p}^{2}\right)}\right. \\
& +\frac{\epsilon \epsilon_{k}+E_{p k}}{\epsilon_{k} E_{p k}\left[\left(\epsilon_{k}+E_{p k}\right)^{2}-E_{p}^{2}\right]}+\frac{2 M^{2}\left(\epsilon_{k}+E_{p k}\right)\left(E_{p}^{2}-M^{2}\right)}{\epsilon_{k} E_{p k} E_{p}^{2}\left[\left(\epsilon_{k}+E_{p k}\right)^{2}-E_{p}^{2}\right]^{2}} \\
& \left.+\frac{\left(\epsilon_{k}+2 E_{p k}\right)\left(E_{p}^{2}-M^{2}\right)\left(2 E_{p}^{2}-M^{2}\right)}{\epsilon_{k} E_{p k} E_{p}^{2}\left[\left(\epsilon_{k}+E_{p k}\right)^{2}-E_{p}^{2}\right]\left(E_{p k}^{2}-E_{p}^{2}\right)}\right\}, \\
\text { "eq. (B.4)" = } & \int_{\mathbf{k}} \mathbb{P}\left\{\frac{1-\epsilon}{E_{p}^{2}}\left(\frac{1}{\epsilon_{k}}-\frac{1}{E_{p k}}\right)-\frac{(1-\epsilon) M^{2}}{2 E_{p}^{2} E_{p k}^{3}}\right. \\
& \left.+\frac{\left[-E_{p}^{2}+\left(\epsilon_{k}+E_{p k}\right)\left(\epsilon_{k}+3 E_{p k}\right)\right] M^{4}}{E_{p}^{2} E_{p k}^{3}\left[\left(\epsilon_{k}+E_{p k}\right)^{2}-E_{p}^{2}\right]^{2}}\right\} .
\end{aligned}
$$


Reducing to a basis of independent structures as indicated in eqs. (B.17)-(B.20) of ref. [28], and carrying out the integrals in the structures with one or two propagators, we obtain

"eq. (B.3)" =

$$
\begin{aligned}
\left\{\int_{K} \mathbb{P}\right. & {\left[2\left(1-\frac{M^{2}}{E_{p}^{2}}\right)\left(\frac{1-\epsilon}{M^{2} \Delta_{K}}-\frac{1-\epsilon}{M^{2} K^{2}}+\frac{1}{\Delta_{K}^{2}}-\frac{\epsilon}{\Delta_{K} \Delta_{K-Q}}\right)\right.} \\
+ & \frac{2 M^{2}}{E_{p}^{2}}\left(\frac{1}{K^{2} \Delta_{P-K}}-\frac{1}{\Delta_{K} \Delta_{K-Q}}\right) \\
& \left.\left.+\left(1-\frac{M^{2}}{E_{p}^{2}}\right) \frac{4 M^{2}}{K^{2} \Delta_{P-K}^{2}}+\left(1-\frac{M^{2}}{E_{p}^{2}}\right) \frac{4\left(2 E_{p}^{2}-M^{2}\right)}{K^{2} \Delta_{P-K} \Delta_{P-K-Q}}\right]\right\}_{p_{0}=i E_{p}, Q=\left(2 i E_{p}, \mathbf{0}\right)}+\mathcal{O}(\epsilon) \\
= & \frac{1}{8 \pi^{2}}\left[\frac{M^{2}}{E_{p}^{2}}\left(1+\frac{p}{E_{p}} \ln \frac{E_{p}+p}{E_{p}-p}\right)-1\right] \\
& +\left(1-\frac{M^{2}}{E_{p}^{2}}\right) \int_{\mathbf{k}} \mathbb{P}\left\{-\frac{M^{2}}{E_{p k}}\left[\frac{1}{2 \epsilon_{k}\left(\epsilon_{k}+E_{p}\right) \Delta_{++}^{2}}+\frac{1}{2 \epsilon_{k}\left(\epsilon_{k}-E_{p}\right) \Delta_{-+}^{2}}-\frac{1}{\left(\epsilon_{k}^{2}-E_{p}^{2}\right) E_{p k}^{2}}\right]\right. \\
& \left.-\frac{2 E_{p}^{2}-M^{2}}{2 \epsilon_{k}^{2} E_{p} E_{p k}}\left(\frac{1}{\Delta_{+}}+\frac{1}{\Delta_{-}}-\frac{1}{\Delta_{++}}+\frac{1}{\Delta_{-+}}\right)\right\}+\mathcal{O}(\epsilon),
\end{aligned}
$$

"eq. (B.4)" =

$$
\begin{aligned}
& \left\{\int_{K} \mathbb{P}\left[\frac{2(1-\epsilon)}{E_{p}^{2}}\left(\frac{1}{K^{2}}-\frac{1}{\Delta_{K}}\right)+\frac{2 M^{2}}{E_{p}^{2} K^{2} \Delta_{P-K}}-\frac{2(2-\epsilon) M^{2}}{E_{p}^{2} \Delta_{K}^{2}}\right]\right\}_{p_{0}=i E_{p}, Q=\left(2 i E_{p}, \mathbf{0}\right)}+\mathcal{O}(\epsilon) \\
& =\frac{3}{8 \pi^{2}} \frac{M^{2}}{E_{p}^{2}}+\mathcal{O}(\epsilon) .
\end{aligned}
$$

In the k-integral of eq. (B.5) the angular integration can be carried out, which leads to the vacuum part of eq. (4.12); eq. (B.6) in turn yields the vacuum part of eq. (4.13). Note that both eq. (B.5) and (B.6) contain a counterterm contribution from eq. (4.3) and are therefore finite in the ultraviolet regime (large $k$ ).

\section{References}

[1] S. Weinberg, Entropy generation and the survival of protogalaxies in an expanding universe, Astrophys. J. 168 (1971) 175 [INSPIRE].

[2] S. Weinberg, Gravitation and cosmology, Wiley, New York U.S.A. (1972).

[3] P.B. Arnold, C. Dogan and G.D. Moore, The bulk viscosity of high-temperature QCD, Phys. Rev. D 74 (2006) 085021 [hep-ph/0608012] [INSPIRE].

[4] G.D. Moore and O. Saremi, Bulk viscosity and spectral functions in QCD, JHEP 09 (2008) 015 [arXiv:0805.4201] [INSPIRE].

[5] E. Lu and G.D. Moore, The bulk viscosity of a pion gas, Phys. Rev. C 83 (2011) 044901 [arXiv: 1102.0017] [INSPIRE].

[6] G. Torrieri, B. Tomášik and I. Mishustin, Bulk viscosity driven clusterization of quark-gluon plasma and early freeze-out in relativistic heavy-ion collisions, Phys. Rev. C 77 (2008) 034903 [arXiv:0707.4405] [inSPIRE]. 
[7] A. Monnai and T. Hirano, Effects of bulk viscosity at freezeout, Phys. Rev. C 80 (2009) 054906 [arXiv: 0903.4436] [INSPIRE].

[8] K. Rajagopal and N. Tripuraneni, Bulk viscosity and cavitation in boost-invariant hydrodynamic expansion, JHEP 03 (2010) 018 [arXiv:0908.1785] [INSPIRE].

[9] H. Song and U.W. Heinz, Interplay of shear and bulk viscosity in generating flow in heavy-ion collisions, Phys. Rev. C 81 (2010) 024905 [arXiv:0909.1549] [INSPIRE].

[10] D. Bödeker and M. Laine, Sommerfeld effect in heavy quark chemical equilibration, JHEP 01 (2013) 037 [arXiv:1210.6153] [INSPIRE].

[11] A. Andronic, P. Braun-Munzinger, K. Redlich and J. Stachel, Statistical hadronization of heavy quarks in ultra-relativistic nucleus-nucleus collisions, Nucl. Phys. A 789 (2007) 334 [nucl-th/0611023] [INSPIRE].

[12] STAR collaboration, M. Mustafa, Measurements of non-photonic electron production and azimuthal anisotropy in $\sqrt{s_{N N}}=39,62.4$ and $200 \mathrm{GeV}$ Au+Au collisions from STAR at RHIC, Nucl. Phys. A 904-905 (2013) 665c [arXiv:1210.5199] [INSPIRE].

[13] ALICE collaboration, G. Ortona, Open-charm meson elliptic flow measurement in $\mathrm{Pb}-\mathrm{Pb}$ collisions at $\sqrt{s_{N N}}=2.76 \mathrm{TeV}$ with ALICE at the LHC, arXiv:1207.7239 [INSPIRE].

[14] S. Caron-Huot and G.D. Moore, Heavy quark diffusion in perturbative QCD at next-to-leading order, Phys. Rev. Lett. 100 (2008) 052301 [arXiv:0708.4232] [INSPIRE].

[15] S. Caron-Huot, M. Laine and G.D. Moore, A way to estimate the heavy quark thermalization rate from the lattice, JHEP 04 (2009) 053 [arXiv:0901.1195] [INSPIRE].

[16] H.B. Meyer, The errant life of a heavy quark in the quark-gluon plasma, New J. Phys. 13 (2011) 035008 [arXiv: 1012.0234] [InSPIRE].

[17] A. Francis, O. Kaczmarek, M. Laine and J. Langelage, Towards a non-perturbative measurement of the heavy quark momentum diffusion coefficient, PoS (LATTICE 2011) 202 [arXiv: 1109.3941] [INSPIRE].

[18] D. Banerjee, S. Datta, R. Gavai and P. Majumdar, Heavy quark momentum diffusion coefficient from lattice QCD, Phys. Rev. D 85 (2012) 014510 [arXiv:1109.5738] [INSPIRE].

[19] G.D. Moore and D. Teaney, How much do heavy quarks thermalize in a heavy ion collision?, Phys. Rev. C 71 (2005) 064904 [hep-ph/0412346] [INSPIRE].

[20] H. van Hees, M. Mannarelli, V. Greco and R. Rapp, Nonperturbative heavy-quark diffusion in the quark-gluon plasma, Phys. Rev. Lett. 100 (2008) 192301 [arXiv:0709.2884] [INSPIRE].

[21] M. Laine, G.D. Moore, O. Philipsen and M. Tassler, Heavy quark thermalization in classical lattice gauge theory: lessons for strongly-coupled QCD, JHEP 05 (2009) 014 [arXiv:0902.2856] [INSPIRE].

[22] F. Riek and R. Rapp, Quarkonia and heavy-quark relaxation times in the quark-gluon plasma, Phys. Rev. C 82 (2010) 035201 [arXiv:1005.0769] [INSPIRE].

[23] W. Alberico et al., Heavy flavors in AA collisions: production, transport and final spectra, Eur. Phys. J. C 73 (2013) 2481 [arXiv:1305.7421] [INSPIRE].

[24] C. Herzog, A. Karch, P. Kovtun, C. Kozcaz and L. Yaffe, Energy loss of a heavy quark moving through $\mathcal{N}=4$ supersymmetric Yang-Mills plasma, JHEP 07 (2006) 013 [hep-th/0605158] [INSPIRE]. 
[25] S.S. Gubser, Drag force in AdS/CFT, Phys. Rev. D 74 (2006) 126005 [hep-th/0605182] [INSPIRE].

[26] J. Casalderrey-Solana and D. Teaney, Heavy quark diffusion in strongly coupled $\mathcal{N}=4$ Yang-Mills, Phys. Rev. D 74 (2006) 085012 [hep-ph/0605199] [INSPIRE].

[27] G. Torrieri and J. Noronha, Flavoring the quark-gluon plasma with charm, Phys. Lett. B 690 (2010) 477 [arXiv: 1004.0237] [INSPIRE].

[28] Y. Burnier and M. Laine, Massive vector current correlator in thermal QCD, JHEP 11 (2012) 086 [arXiv:1210.1064] [INSPIRE].

[29] S.L. Adler, J.C. Collins and A. Duncan, Energy-momentum-tensor trace anomaly in spin 1/2 Quantum Electrodynamics, Phys. Rev. D 15 (1977) 1712 [InSPIRE].

[30] J.C. Collins, A. Duncan and S.D. Joglekar, Trace and dilatation anomalies in gauge theories, Phys. Rev. D 16 (1977) 438 [inSPIRE].

[31] M. Laine and Y. Schröder, Quark mass thresholds in QCD thermodynamics, Phys. Rev. D 73 (2006) 085009 [hep-ph/0603048] [INSPIRE].

[32] RBC-BIELEFELD collaboration, M. Cheng, Charm quarks and the QCD equation of state, PoS(LATTICE 2007) 173 [arXiv:0710.4357] [inSPIRE].

[33] C. DeTar et al., QCD thermodynamics with nonzero chemical potential at $N_{t}=6$ and effects from heavy quarks, Phys. Rev. D 81 (2010) 114504 [arXiv:1003.5682] [INSPIRE].

[34] S. Borsányi et al., The QCD equation of state and the effects of the charm, PoS(LATTICE 2011) 201 [arXiv: 1204.0995] [INSPIRE].

[35] D. Bödeker and M. Laine, Heavy quark chemical equilibration rate as a transport coefficient, JHEP 07 (2012) 130 [arXiv: 1205.4987] [INSPIRE].

[36] M. Laine, M. Vepsäläinen and A. Vuorinen, Ultraviolet asymptotics of scalar and pseudoscalar correlators in hot Yang-Mills theory, JHEP 10 (2010) 010 [arXiv:1008.3263] [INSPIRE].

[37] M. Laine, A. Vuorinen and Y. Zhu, Next-to-leading order thermal spectral functions in the perturbative domain, JHEP 09 (2011) 084 [arXiv:1108.1259] [INSPIRE].

[38] H.B. Meyer, The bulk channel in thermal gauge theories, JHEP 04 (2010) 099 [arXiv: 1002.3343] [INSPIRE].

[39] H.-T. Ding et al., Charmonium properties in hot quenched lattice QCD, Phys. Rev. D 86 (2012) 014509 [arXiv: 1204.4945] [INSPIRE].

[40] M. Nishimura and Y. Schröder, IBP methods at finite temperature, JHEP 09 (2012) 051 [arXiv: 1207.4042] [INSPIRE].

[41] Particle Data Group collaboration, J. Beringer et al., Review of particle physics (RPP), Phys. Rev. D 86 (2012) 010001 [INSPIRE].

[42] T. van Ritbergen, J.A.M. Vermaseren and S.A. Larin, The four loop $\beta$-function in Quantum Chromodynamics, Phys. Lett. B 400 (1997) 379 [hep-ph/9701390] [INSPIRE].

[43] J.A.M. Vermaseren, S.A. Larin and T. van Ritbergen, The four loop quark mass anomalous dimension and the invariant quark mass, Phys. Lett. B 405 (1997) 327 [hep-ph/9703284] [INSPIRE]. 
[44] W. Florkowski and B.L. Friman, Spatial dependence of the finite temperature meson correlation function, Z. Phys. A 347 (1994) 271 [INSPIRE].

[45] H.B. Meyer, Energy-momentum tensor correlators and spectral functions, JHEP 08 (2008) 031 [arXiv:0806.3914] [INSPIRE].

[46] S. Caron-Huot, Asymptotics of thermal spectral functions, Phys. Rev. D 79 (2009) 125009 [arXiv: 0903.3958] [INSPIRE].

[47] D.J. Broadhurst, J. Fleischer and O.V. Tarasov, Two-loop two-point functions with masses: asymptotic expansions and Taylor series, in any dimension, Z. Phys. C 60 (1993) 287 [hep-ph/9304303] [INSPIRE].

[48] Y. Burnier, M. Laine and M. Vepsäläinen, Heavy quark medium polarization at next-to-leading order, JHEP 02 (2009) 008 [arXiv: 0812.2105] [INSPIRE].

[49] P. Petreczky and D. Teaney, Heavy quark diffusion from the lattice, Phys. Rev. D 73 (2006) 014508 [hep-ph/0507318] [InSPIRE].

[50] Y. Burnier and M. Laine, Temporal mesonic correlators at NLO for any quark mass, arXiv:1310.6124 [INSPIRE]. 